investigation has been continued over a long period of years into various aspects of the problem, including particularly the occupational incidence of the disease, with special reference to farming. It is hoped that the results of this work on a series of 389 cases of disseminated sclerosis will shortly be embodied in a paper by a member of my staff.

Your annotation refers optimistically to the prospects of discovering the cause and prevention of this illness. At the moment I personally feel that the detection of the mode of transmission is the next most valuable step that could be made, and there is a reasonable prospect that we are approaching this state of knowledge. In 1923 I made the following statement (Glasg. med. J., 1923, 100, 290): "A still further advance may then be hoped for in the detection of the mode of transmission and prevention of infection in the individual."

It cannot be assumed that the causal agent of disseminated sclerosis will produce pathological changes in the nervous system of susceptible animals identical with those found in the human subject. Equally it cannot be assumed that infection by the same agent will necessarily result in identical tissue reaction in animals of different species. As an example of this important general principle, the causative agent of "louping ill " is known to produce a separate histological picture in sheep, pigs, and mice (Brownlee, A., and Wilson, D. R., J. comp. Path., 1932, 45, 67). Past experience has shown that this is an essential difficulty in attempting to transmit disseminated sclerosis experimentally to animals. - I am, etc.,

Glasgow.

Douglas K. Adams.

\section{Treatment of Varicose Veins}

SiR,-Prof. A. M. Boyd and Mr. D. J. Robertson (Sept. 20, p. 452) discuss some of the causes of the unsatisfactory results in the treatment of varicose veins. They also illustrate their article with some very interesting phlebograms. With regard to the incidence of the condition, the authors state that $95 \%$ of patients attending civilian varicose vein clinics are women. This was certainly not the case when I had the vein clinic at St. Bartholomew's Hospital, where I found that approximately onequarter of my cases were among men, and this figure was borne out by my private cases. I cannot agree with the suggestion that varicose veins seldom cause serious discomfort. The condition invariably develops very gradually and the patient gets used to the increasing heaviness in the limbs. Severe pain is certainly not a common feature, but the discomfort and disability are great and are aggravated by conditions which involve much standing. At the same time, the degree of discomfort is not necessarily related to the actual size or extent of the veins.

Recurrence of varicosities after treatment may mean a failure to obliterate all the incompetent veins; it may mean a gradual recanalization of veins which were obliterated at the time of treatment; or it may mean the gradual development of varicosities in other veins. My own experience has led me to an operation-a combined excision and ligation-with at least two, and possibly three, incisions in the thigh and with two incisions in the calf, with a further large popliteal incision where the external saphenous vein is involved. Subsequently, any small remaining veins are treated by injections. At the same time I do not believe that the mere ligation operation at sites of valvular incompetence of communicating veins will secure obliteration of extensive varicosities, even when combined with simultaneous injections. By suitably planning the direction of incisions it is possible to use the flexure lines of the skin, to get adequate exposure of considerable lengths of the veins, and to avoid incisions in the long axis of the limbs. In one week recently I saw three patients all of whom had had recent ligation. and injection operations at the groins, and all of whom showed complete incompetence of the whole saphenous systems right up to the level of the operation scars. Neither the symptoms nor the signs suggested that there had been any appreciable alteration as the result of the treatment.

Operative experience also suggests that by far the most important deep communicating vein is the one situated at the junction of the middle and lower third of the leg as a communication between the internal saphenous vein or one of its branches and the deep veins. Certainly, from the point of view of local symptoms, the failures of injection treatment, and the local complications such as eczema, ulceration, etc., this is the branch which is so often responsible for a reflux of blood into the superficial veins. The presence of this vein can often be inferred from the physical signs but cannot be proved until operation is carried out.

Prof. Boyd states that between October, 1942, and February, 1943, he saw 12 patients with deep thrombosis following injection treatment in the Army. I am not quite clear from the context whether these patients had had a combination of operation and injections or injections alone. In my own series of approximately 2,000 varicose vein cases $I$ have never seen this complication following operation, operation and subsequent injections, or injections alone. On the other hand, whatever form of treatment is adopted, there are cases which show temporary and transient oedema, particularly on the inner side of the foot and ankle, for some weeks after treatment. This is much more likely to occur where there is much subcutaneous tissue, where operations have been extensive, or thrombosis has been widespread, but I have never known this oedema persist. The proper treatment, of course, is to control the oedema with external support, and, as lymphatic function is re-established, gradually to lessen the external support. In view of what Prof. Boyd says about the reaction of men in the Army to varicose veins, I am wondering if some of the deep thrombosis cases were really post-treatment oedemas and not deep thromboses at all. The presence of an abdominal collateral circulation would be conclusive. The authors suggest that this complication of deep thrombosis may have been due to the passage of injection fluid into the femoral veins, where the circulation may be sluggish. I am sure that some of the sclerosing fluid must get into the deep veins following injections, but there are two comments $I$, would like to make in this connexion. The first is that the more normal the structure of the vein wall, the greater the difficulty in getting thrombosis with sclerosants. And, secondly, that in those patients who have a slight sensitivity to quinine the drug is tasted within approximately 15 to 20 seconds of injection of the peripheral veins of the lower limb with the patient lying down.

London, W.1.

Reginald T. Payne.

SIR,-Prof. A. M. Boyd and Mr. D. J. Robertson's most interesting article on the above subject (Sept. 20, p. 452) calls attention to the unsatisfactory results of the treatment of the all too common varix. I feel that their conclusions tend to advise a standard treatment of varicosities needing surgical care. They state: "Ligation is required at four sites ..." "High retrograde injection of large quantities of ... sclerosant is dangerous," etc. I submit that each case of severe varices needs a careful assessment and that varied treatments are called for according to the findings at the time of examination. The Ochsner-Mahorner tourniquet test will tell the operator the site of the incompetent communicating veins. Four standard sites of ligation will then not be found necessary in the majority of cases. For instance, in a large series of cases these surgeons found that it was only necessary to ligate the saphenous below the knee in $2 \%$ of patients.

Again, to condemn the use of retrograde injection of a sclerosant into the distal portion of the internal saphenous divided at the fossa ovalis is far too sweeping a statement. If the patient has suffered from an antecedent deep-vein thombophlebitis care should be observed regarding the use of any sclerosant. Has there been a recent superficial phlebitis? In these cases use no sclerosant if a severe reaction is to be avoided. There are other indications for the modification of the type and amount of sclerosant to be used. The "mid-Hunter blow-out" (aptly named by various American surgeons) is, as proved by Prof. Boyd and Mr. Robertson, responsible for the admission of much sclerosant into the deep system. The careful use of a Stevenson needle (or one of its modifications) can mitigate this risk. Again, hypertonic saline in the hands of Harold Dodd has satisfied him and many others that damage to the normal intima does not occur.

In a short letter $I$ can but endeavour to point out that standardized treatments and dogmatic statements cannot be applied to the vagaries of the varix. It is seldom that two cases present exactly the same problem. The authors of the article under discussion are, however, to be congratulated on calling attention to the fact that the treatment of varices can be 